\title{
Evidence-based Complementary and Alternative Medicine
}

\section{List of reviewers}

We thank the scientists and scholars listed here who have given anonymously of their time in order to make CAM more rigorous.

Without your astute comments, an evidence base for CAM would remain a dream. We have starred the names of those who have demonstrated great support in reviewing five or more papers.

\begin{tabular}{|c|c|c|}
\hline Y.H. Abdel-Wahab & Y.Y. Bilto & A. Cuerrier \\
\hline M. Abdollahi & D. Blohm & M. Curran \\
\hline K. Abe & C. Bodinet & A.C. da Nobrega \\
\hline T. Abo & K. Boini & Y. Dai \\
\hline M. Abolhassani & J. Bomser & E. Dannecker \\
\hline D. Abrams & A. Bomzon & H.R. Das \\
\hline J. Adams* & L. Bonamin & A. de la Taille \\
\hline A.C. Adebajo & H. Boon & A.J. De Lucca \\
\hline S. Adesegun & F. Borrelli & H. Dejian \\
\hline A. Adetutu & B. Brinkhaus & M.L. Dell \\
\hline O. Akinloye & J.B. Brissaud & T. Devaki \\
\hline Z. Al-Ani & J. Buckle & S.P. Dhanabal \\
\hline N. Al-Waili & E.J. Buenz & B. Diamond \\
\hline B.H. Ali & H. Buttar & S. Dieterle \\
\hline M. Ali & H. Cannizzaro & C. Dileo \\
\hline M.S. Ali & P. Canter & B. Dinda \\
\hline T. Alraek & J. Capodice & E. DiNucci \\
\hline M. Alvarez de Sotomayor & S. Caprilli & T. Dorai \\
\hline A. Amin & C. Carpenter* & J.J. Dugoua \\
\hline V.N. Anisimov & F. Ceccherelli & T. Dunning \\
\hline V. Arndt & A.O. Chan & M. Eddouks \\
\hline A. Aroor & P. Chan & E. Elisabetsky \\
\hline H. Azaizeh & D. Chang & E. Ercolano \\
\hline H. Bae* & I.M. Chang & E. Ernst* \\
\hline M. Baecker & K.M. Chang & L. Eshkevari \\
\hline L.B. Balsam & S.T. Chang & A. Esteban \\
\hline V. Bankova & M. Chaplin & S. Estrada-Soto \\
\hline P. Barlas & M.R.S. Chaturvedi & E. Farombi \\
\hline V. Barnes & A. Chaudhry & Z. Fatehi-Hassanabad \\
\hline N. Basaran & C.N. Chen & M.O. Fatope \\
\hline D. Baxter & J.T. Cheng* & A. Felicioli \\
\hline G. Beauchamp & H.Y. Cheung & C. Fernandez-de-las-penas \\
\hline C. Beedie & F. Chiappelli* & D.J.S. Fernando \\
\hline S. Beider & C.C. Chiu & T. Field \\
\hline P. Bellavite* & M.K. Cho & P. Fisher \\
\hline E. Ben-Arye & K. Chopra & J. Flowers \\
\hline J. Berman & C.C. Chou & G. Franz \\
\hline W. Bernard & A. Cider & S. Furukawa \\
\hline F. Bersani & P. Clifton & J. Gallo \\
\hline S. Bertisch & J. Consigli & A. Galvez \\
\hline P. Bewes & K. Cooley & S.E. Geller \\
\hline K.K. Bhutani & O. Coskun & P. Gerbarg \\
\hline L. Bielory & A. Cossarizza & A. Gilani* \\
\hline F.C. Biley & M. Coyle & C.M. Giovanardi \\
\hline
\end{tabular}


G. Gobe

R.K. Goel

B. Gold

J. Gold

M. Goldstein

H. Goto

D. Grande

L. Grazzi

R. Greenfield

E. Grossi

A. Gugliucci

I. Guler

S.T. Gura

G.W. Hacker

P. Haddad

J. Halpern

M. Hamann

A. Hankey*

H.P. Hanssen

T. Haqqi

R. Harris

M. Heinrich

Y. Henrotin

C.J. Henry

G. Hernandez

M. Herrera-Ruiz

H. Hideaki

H. Hikiami

C. Hioki

J.W. Ho

Y.K. Hong

V. Hopwood

H. Hosseinzadeh

P. Houghton*

M.C. Houston

M.T. Hsieh

J. Hsu

Y.T. Huang

C. Hui

P. Hummel

M. Hyland

J. Imanishi*

N. Inagaki*

$M$. Inoue

M. Inoue

M. Iranshahi

M. Irwin

M. Ishii*

M. Italiano

S. Itohara

J.C. Ives

G.C. Jagetia

J. Jamal*

C. Johnston

J. Jordan

H. Joshi*
M. Justiss

S. Kadota

B. Kaeffer

C. Kamei

J. Kang

M. Kanter

K. Kaphle*

G. karimi

M. Karst

S. Katz

M. Kaur

B. Kavoussi

K. Kawakita*

K. Kawase

C. Kelly

J. Kelly

A. Khalil

M.R. Khan

M.L. Khatri

A. Khuda-Bukhsh

P.M. Kidd

R. Kikkawa

C.J. Kim

G.Y. Kim

S.K. Kim

S.H. Kim

Y.S. Kim

I. Kirman

A. Kleindeist

K.M. Ko

H. Kobayashi*

W. Koenig

B.H. Koh

K. Komiyama

K. Korotkov

I. Kosalec

R. Krochmal

A. Kumar

C. Kumar

E. Kumarnsit

G. Kuttan

J. Lackner

L. Lao

M. Leal

D.Y. Lee

E.J. Lee

M.S. Lee

S.S. Lee

T.K. Lee

S. Leite*

P.C. Leung

G. Lewith

E. $\mathrm{Li}$

W.M. Li

S. Lim

J.K. Lin
Z.B. Lin

K. Linde

U. Lindequist

L. Lippiello

L. Liu

T. Liu

S. Lo

D. Lonsdale

A. $\mathrm{Lu}$

Y.M. Lucisano-Valim

A. Lugasi

T. Lundeberg

S.K. Lutgendorf

P. Lys de Medeiros

C.M. Ma

S.X. Ma

V.G. Macefield

C. Malemud

R. Manohar

I. Mansi

P. Mansky

M.S. Mantovani

E. Marie

M.S. Markov

F. Marotta*

P. Matravers

D. Matsui

J. Mayer

F. McCartney

A. McDonnell

D. Mehta

M. Mejia

H.Q. Miao

A. Michalsen

J. Milei

L. Milgrom

S. Miller

E. Mills

L. Min

E. Minelli*

P. Minodier

S.H. Mishra

T. Miyata

T. Mizutani

A. Molassiotis

G. Montgomery

L. Monzote

S. Moolasam

D. Morre

E.L. Morris

Y. Motoo*

C. Moyer

T. Murayama

B. Musicki

C. Myers

T. Mynott 
H. Nagai

Y. Naito

R.A. Najim

S. Nanduri

F. Nepveu

E. Nestmann

C.W. Newman

T.B. $\mathrm{Ng}$

O. Ngassapa

T. Nicolai

H. NISHIDA

M. Noguchi

T. Oegema

J. Ogden

C. Ohkubo

S. Ohnishi

H. Okamoto*

K.S. Olah

Jose. Olalde

D. Orme-Johnson

D. Owen

S.C. Pak

O.S. Palsson

E. Papadimitriou

H. Park

J. Park

Y.M. Park

M. Parle

S. Pathak

M. Patil

Y. Perez Teran

R. Perumal Samy

S. Phipps

K. Pilkington

A.L.B. Pinheiro

L. Pinto

N. Pitsikas

J. Post-White

B. Potrata

P. Pramyothin

K. Premkumar

P. Pushpangadan

B. Qin

M. Rabbani

A. Radloff

B. Rajeshkumar

M. Rao

S. Rastogi

M. Ravindranath*

A. Ray

P.H. Reddy

G. Rees

J.C. Reeser

V.E. Reeve

M. Renis

L. Rice
M. Rittman

N. Rizzo-Roberts

P. Roberti di Sarsina*

R. Roesler

I.A. Rorsman

E. Ross

P. Rosted

J. Rubio

L. Rutten

D. Ryan

B. Saad

M. Saleem

O. Santos da Silva

S. Saraf

K. Satyamoorthy

R. Sawhney

A. Scheve

G. Schmeda-Hirschmann

D. Seely

M. sekar

S. Selvan

K. Sembulingam

H. Senjyu

Z.A. Shah

B. Shaikh

R.K. Sharma

R. Sharma

V.K. Sharma

X.C. Shen

S. Shetty

J. Shi

S. Shimizu

D.H. Shin

A. Shirwaikar

G. Shishu

A. Shmueli

D. Sibbritt

C.M. Siedentopf

J. Sim

J. Sim

S. Simjeet

R. Singh

P. Sipos

G. Sivakumar

D. Sliva

K. Sluka

D.H. Sohn

E. Speroni

G. Spiteller

P. Stamets

R. Staud

E. Stener-Victorin

S. Stepanovic

J. Stephens

B. Sternlieb

J. Still
A. Stone

G. Strauss-Blasche

M. Stuber

N. Sukul

G.C. Sun

M.J. Sun

Z.J. Sun

K. Sundram

L. Sung

C. Suthisisang

N. Suzuki

F. Sze

J.C. Szeles

R. Takahashi

T. Takahashi

K. Takeda

L. Tan

H. Tanaka

T. Tanaka

J. Tang

Y.L. Tang

A. Tavridou

S. Taweechaisupapong

A. Taylor

M. Teixeira

P. Thakurdesai

A. Thurneysen

S. Tirmzi

S. Tjen-A-Looi

T. Togo

C. Tohda

N. Tomita

M. Tournaire

D. Trauner

Y. Tripathi

J. Tsao*

H. Tsukayama

S.J. Uddin

P. Unschuld

T. Ushiroyama

T. Usichenko*

A. Uzel

G. Vaidyanathan

P. Varalakshmi

J. Vas

P. Vayalil

M. Venglar

C. Ventura*

R.J. Verma

H. Vernon

G. Vidotti

I. Villasenor

P. Visen

H. Wago

I. Wakayama

R. Walker 


$\begin{array}{lll}\text { K. Walton } & \text { Y. Wong } & \text { D. Yew } \\ \text { B.G. Wang } & \text { S. Wongkham } & \text { A. Yim } \\ \text { W.K. Wang } & \text { O. Wongmekiat } & \text { C.L. Yu } \\ \text { X. Wang } & \text { G.C. Wu } & \text { M. Zander } \\ \text { Z. Wang } & \text { X.E. Wu } & \text { P. Zanoli } \\ \text { D. Wardell } & \text { T. Xia } & \text { M. Zanoni } \\ \text { G. Watal } & \text { B.Z. Xiang* } & \text { N. Zarkovic } \\ \text { L. Watkins } & \text { T. Xiang } & \text { M. Zasloff } \\ \text { D. Webster } & \text { H. Xu } & \text { B. Zee } \\ \text { C. Weze } & \text { H. Yamada* } & \text { L. Zeltzer } \\ \text { A. White } & \text { N. Yamaguchi* } & \text { P. Zeltzer } \\ \text { P. White } & \text { M. Yamamoto } & \text { G.G. Zhang } \\ \text { R. White-Traut } & \text { C.H. Yang } & \text { L. Zhang } \\ \text { B. Williams } & \text { C. Yang } & \text { C. Zhao } \\ \text { R. Williams } & \text { J. Yang } & \text { Z. Zhao } \\ \text { T. Williams } & \text { Y. Yazawa } & \text { Y. Zhou } \\ \text { E. Wollenweber } & \text { R. Yazdanparast } & \\ \text { S. Wong } & \text { G. Yeh } & \end{array}$




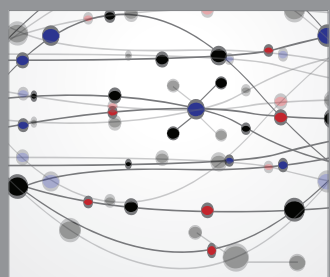

The Scientific World Journal
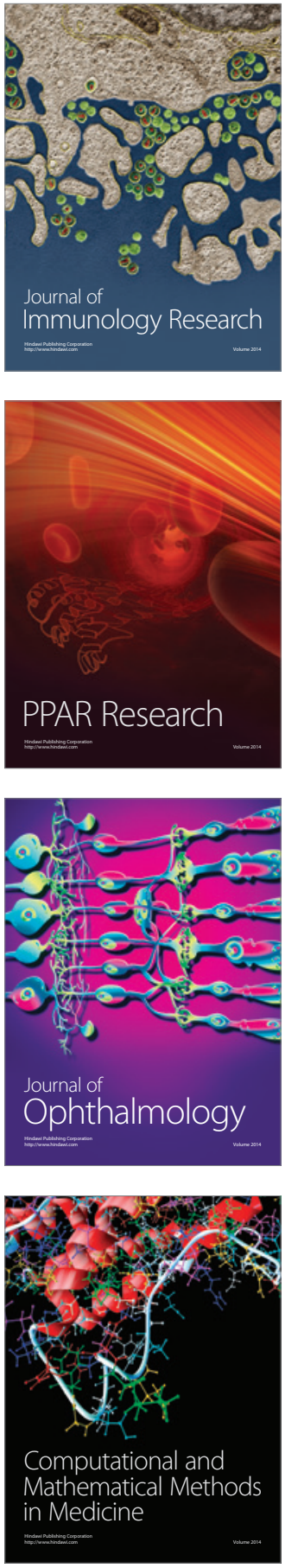

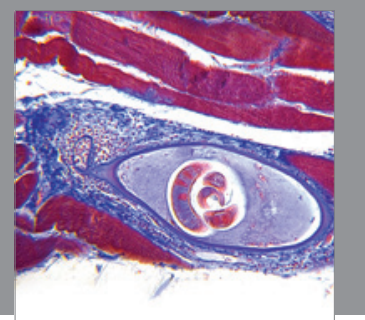

Gastroenterology

Research and Practice
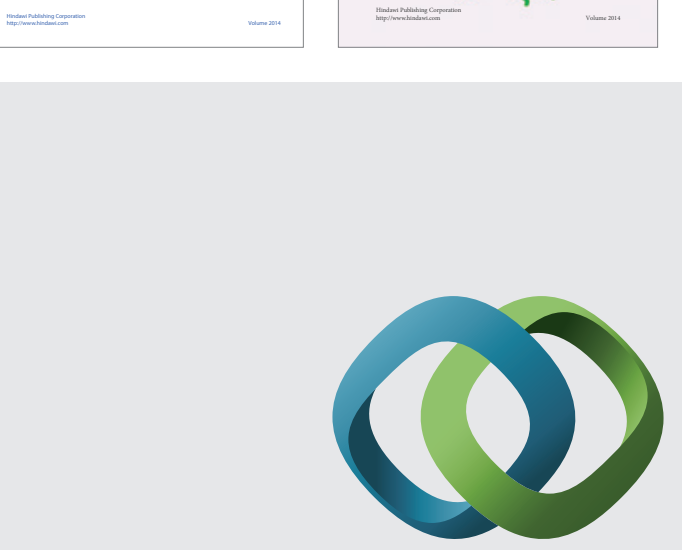

\section{Hindawi}

Submit your manuscripts at

http://www.hindawi.com
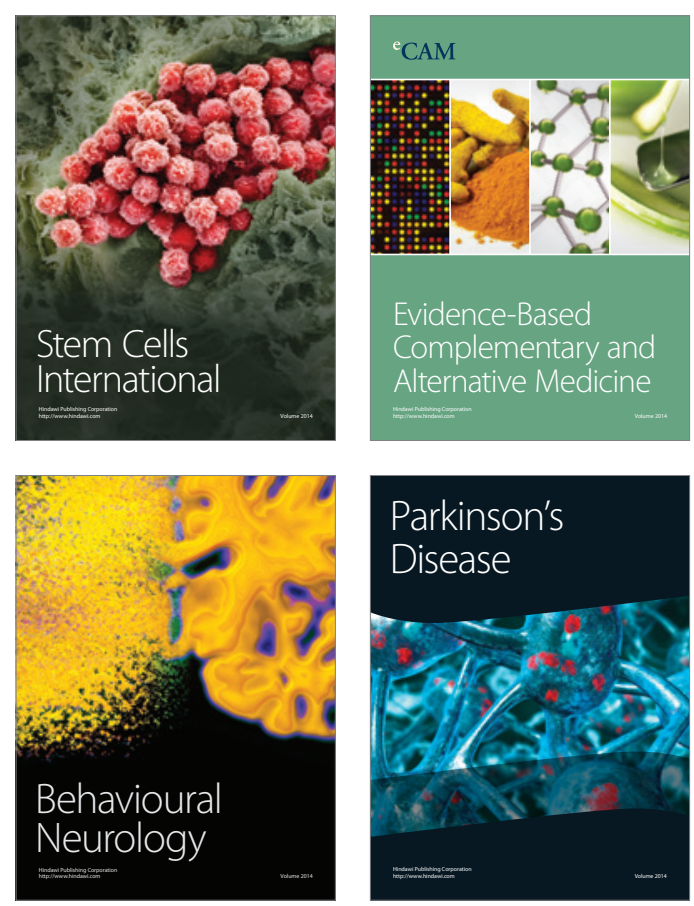

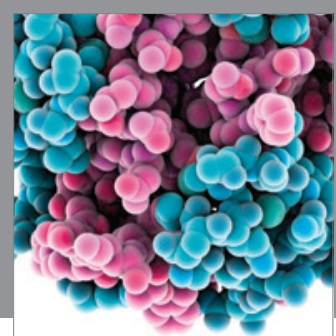

Journal of
Diabetes Research

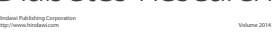

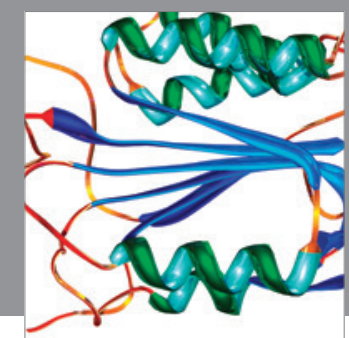

Disease Markers
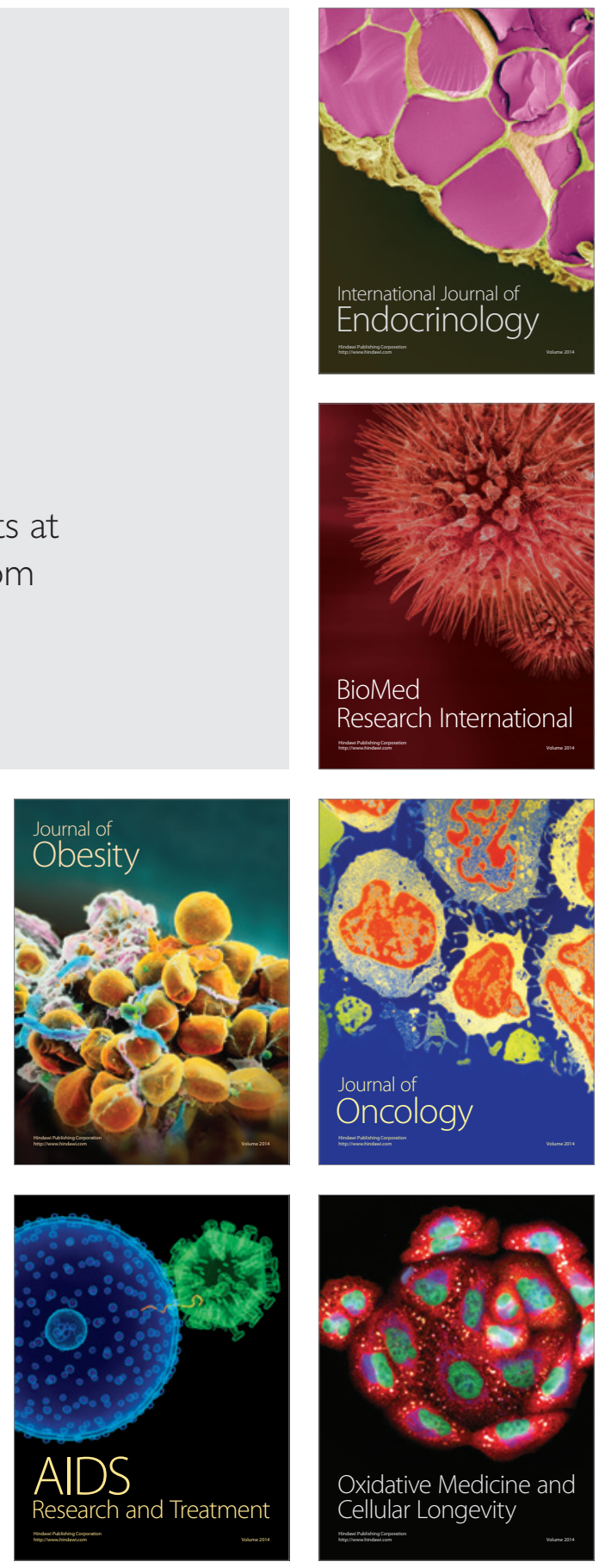